\section{Uso de linkage entre diferentes bases de dados para qualificação de variáveis do Sinan-TB e a partir de regras de scripting}

\author{
Use of database linkage and scripting rules to \\ upgrade variables in the Sinan-TB database
}

\section{Uso del linkage entre diferentes bases de datos para la cualificación de variables del Sinan-TB, a partir de reglas de scripting}

\section{Resumo}

O Sistema de Informação de Agravos de Notificação (Sinan) é a principal ferramenta utilizada pelos programas de controle da tuberculose (TB), para avaliar as ações de controle e a taxa de incidência da doença. Para tal, fazse necessário que os dados provenientes desse sistema sejam, dentre outros, confiáveis e acurados. Dessa forma, o objetivo deste estudo foi qualificar as variáveis tipo de entrada, situação de encerramento, teste para HIV, agravo associado aids e diabetes. Foi realizado um linkage entre a base de dados do Sinan-TB, do Sistema de Informações sobre Mortalidade (SIM) e a base única da aids do Município do Rio de Janeiro, Brasil. Os critérios para qualificação das variáveis estão fundamentados em materiais técnicos sobre a TB e sobre o Sinan, e foram implementados em um script em Structured Query Language (SQL). Observou-se um aumento de $115 \%$ do abandono de tratamento decorrente da diminuição das transferências, dos registros sem encerramento e de pacientes encerrados por cura com menos de 150 dias. Houve acréscimo de $2,4 \%$ de registros com agravo associado diabetes na categoria sim, após utilizada a informação contida no SIM. Para as variáveis teste para HIV e agravo associado à aids o acréscimo foi de 5,3\% e 8,7\%, respectivamente, com a consideração da informação contida na base da aids. Concluiu-se que a qualificação da base de dados do Sinan-TB, valendo-se da integração com outros sistemas de informação, aprimorou a qualidade da informação para a tomada de decisão para o controle da TB.

Sistemas de Informação; Registro Médico Coordenado; Tuberculose; Confiabilidade dos Dados; Bases de Dados
QUESTÕES METODOLÓGICAS

METHODOLOGICAL ISSUES

Marli Souza Rocha 1

Gisele Pinto de Oliveira 1

Luis Carlos Torres Guillen 1

Claudia Medina Coeli 1

Valéria Saraceni 2

Rejane Sobrino Pinheiro 1

doi: 10.1590/0102-311X00074318

\section{Correspondência}

M. S. Rocha

Instituto de Estudos em Saúde Coletiva, Universidade Federal do Rio de Janeiro.

Av. Horácio Macedo s/n, Próximo a Prefeitura Universitária da UFRJ, Rio de Janeiro, RJ 21944-970, Brasil.

marli.rocha@gmail.com

1 Instituto de Estudos em Saúde Coletiva, Universidade Federal do Rio de Janeiro, Rio de Janeiro, Brasil.

2 Superintendência de Vigilância em Saúde, Secretaria Municipal de Saúde do Rio de Janeiro, Rio de Janeiro, Brasil. 


\section{Introdução}

O Sistema de Informação de Agravos de Notificação - Tuberculose (Sinan-TB) é a principal ferramenta usada pela vigilância dos municípios, estados e Distrito Federal para notificar, investigar, acompanhar e analisar os dados sobre pacientes diagnosticados com TB no Brasil 1. Entretanto, os erros de classificação dos casos são comuns, principalmente, para as variáveis "tipo de entrada" e "situação de encerramento", o que reflete informações equivocadas 2,3. Um estudo realizado em um município da Região Metropolitana do Rio de Janeiro no período de 2006 a 2008, comparou o Sinan-TB com o Livro de Registro de Pacientes e Acompanhamento de Tratamento, e verificou que $16,9 \%$ e $6 \%$ dos casos apresentaram informações discordantes para o encerramento e para a variável tipo de entrada, respectivamente 4 .

Durante o tratamento terapêutico da TB, o paciente pode ser transferido, voluntariamente ou não, para dar continuidade ao tratamento em outra unidade de saúde, sendo, desta forma, notificado mais de uma vez. Em virtude dessa situação, o Sinan-TB dispõe de uma rotina de remoção de duplicidades e de vinculação de registros. Ainda assim, Bartholomay et al. 5 demonstraram que essa rotina não é realizada adequadamente por grande parte dos municípios, acarretando um expressivo número de registros duplicados e com encerramento por transferência. Além disso, periodicamente, são feitas atualizações nas variáveis presentes no Sinan-TB, decorrentes da inclusão de registros e atualização de informações, o que torna esta tarefa sujeita à falha e inconsistências, comprometendo a qualidade da informação.

O Sistema de Informações sobre Mortalidade (SIM) é utilizado para o cálculo da taxa de mortalidade por TB, importante indicador epidemiológico ${ }^{1}$. Ainda assim, essa informação também é coletada pelo Sinan-TB, porém, falhas no critério de saída do paciente ou erro de classificação podem ocorrer, como demonstrado por um estudo de base nacional realizado em 2006, que verificou que, dos óbitos de TB registrados no SIM de pacientes que haviam sido notificados no Sinan-TB no mesmo ano do óbito, 9,2\% apresentaram encerramento cura 3 .

As variáveis "agravo associado aids" e "agravo associado diabetes", por serem categorias de preenchimento não obrigatório no Sinan-TB, apresentam uma baixa completude 6,7,8, não fornecendo a verdadeira associação entre a TB e os agravos citados, os quais são importantes para o prognóstico e para a avaliação do atendimento prestado. A possibilidade de qualificar essas informações no SinanTB, por meio de outros sistemas de informação disponíveis, permite encontrar o valor mais próximo da verdadeira prevalência desses agravos em pessoas com TB.

Considerando a importância e a preocupação com a qualidade dos sistemas de informação para TB, o objetivo deste trabalho é qualificar as variáveis "tipo de entrada" e "situação de encerramento". Essas variáveis são a base para o cálculo dos principais indicadores epidemiológicos e operacionais, a saber, a taxa de incidência e os percentuais de cura e de abandono de tratamento, que são utilizados para avaliar, periodicamente, as ações de controle da TB em todas as esferas de governo. Também foram qualificadas as variáveis "teste para HIV", "agravo associado aids" e "agravo associado diabetes", pois fornecerão uma descrição mais confiável destes agravos.

\section{Método}

O processo de qualificação da base de dados do Sinan-TB foi realizado para os pacientes notificados no Município do Rio de Janeiro, nos anos de 2009 a 2013. Foram usadas as bases de dados do Sinan-TB do Município do Rio de Janeiro de 2004 a 2013; da aids do mesmo município, de 2009 a 2013; e do SIM do Estado do Rio de Janeiro de 2009 a 2013. Foram realizados três procedimentos de linkage para a construção da base de dados do trabalho, descritos a seguir.

Utilizaram-se os dados contidos no SIM para a qualificação do encerramento por óbito e do agravo diabetes, por seus dados serem oriundos de um documento oficial de atestado de óbito, devido à boa cobertura em todo o território nacional e pela baixa proporção de causas mal definidas ${ }^{9}$. Para qualificar a informação de HIV/aids, foi utilizada a base única da aids, por ser composta por dados de diversos sistemas de informações (Sinan, SIM, Sistema de Controle de Exames Laboratoriais - 
SISCEL, e Sistema de Controle Logístico de Medicamentos - SICLOM) o que minimiza a subnotificação de aids no estado.

\section{Deduplicação e vinculação do Sinan-TB}

O primeiro linkage teve como objetivo a eliminação de duplicidades de registros no Sinan-TB, assim como recuperar a história prévia de tratamento do paciente. Foi utilizada a base de dados dos casos notificados no Município do Rio de Janeiro no Sinan-TB entre 2004 e 2013. Para identificar as múltiplas notificações de um mesmo paciente no período, foram realizados dois procedimentos de deduplicação, um por meio de abordagem determinística, seguido de outro usando abordagem probabilística, de acordo com Oliveira et al. 10. A abordagem determinística fundamentou-se em um conjunto de 70 regras construídas valendo-se de pelo menos três critérios baseados nas variáveis de identificação do paciente ou de modificação delas. A abordagem probabilística foi empregada em seguida, para recuperar os pares de registros de um mesmo indivíduo que não tinham sido identificados na etapa anterior. Para isso, foi realizado o procedimento de deduplicação no OpenRecLink (http://reclink.sourceforge. net/) usando-se como ponto de corte o escore 18,3, acima do qual os links encontrados eram considerados pares verdadeiros 10. A classificação dos registros foi realizada por meio dos procedimentos sugeridos por Bierrenbach et al. 11: os registros do mesmo caso, referentes a transferências entre unidades, foram vinculados; foram mantidos os registros do mesmo paciente quando este apresentava história prévia de tratamento, e as duplicidades verdadeiras foram eliminadas. Indivíduos encerrados no Sinan-TB por mudança de diagnóstico não foram incluídos na análise.

\section{Linkage entre o Sinan-TB e a base da aids}

O segundo procedimento de linkage ocorreu entre a base de dados do Sinan-TB contendo os casos notificados no Município do Rio de Janeiro entre 2009 e 2013, deduplicada e vinculada na etapa anterior, e a base da aids, e teve como objetivo qualificar o campo "teste para HIV" e "agravo associado aids" no Sinan-TB. A base da aids era composta pelo produto do linkage entre Sinan-aids, SIM, SISCEL de CD4+/CD8+ e Carga Viral do HIV e SICLOM, conforme procedimento habitual realizado pelo Departamento de Infecções Sexualmente Transmissíveis/AIDS e Hepatites Virais, e descritos em nota técnica da Secretaria de Vigilância em Saúde do Ministério da Saúde 12. A abordagem usada nessa etapa foi a probabilística, composta por 18 passos de blocagem, baseados na combinação do soundex do primeiro nome do paciente e da mãe, soundex do último nome do paciente, ano de nascimento, sexo e município de residência, conforme sugerido por Santos 13. No processo de comparação para o cálculo dos escores estimando quão prováveis dois registros pertenceriam a um mesmo indivíduo, foram utilizados os nomes completos do paciente e da mãe, data de nascimento e sexo. Cada passo de blocagem foi acompanhado de revisão manual.

\section{Linkage do Sinan-TB com o SIM}

O terceiro procedimento de linkage foi realizado entre a base de dados do Sinan-TB de 2009 a 2013 e a base de dados do SIM do Estado do Rio de Janeiro, referente aos anos de 2009 a 2013. Foi usado o mesmo procedimento do item anterior.

Os registros pareados nos procedimentos de linkage anteriores foram acrescentados à base de dados do Sinan-TB.

\section{Script de qualificação do Sinan-TB}

Foram elaborados critérios considerando três diferentes situações para o tempo de tratamento: menor do que 150 dias, entre 150 e 270 dias e maior do que 270 dias (Figuras 1, 2 e 3). O encerramento por cura ocorre quando o paciente completa o período de 180 dias de tratamento da TB com ou sem confirmação bacteriológica 1. Buscou-se verificar possíveis diferenças no tempo de tratamento em função de distintas situações: quando o paciente recebeu medicação no 5 o mês, não retornando para 
Figura 1

Diagrama com os critérios para qualificação da base de dados do Sistema de Informação de Agravos de Notificação - Tuberculose (Sinan-TB) em pacientes com < 150 dias de tratamento.

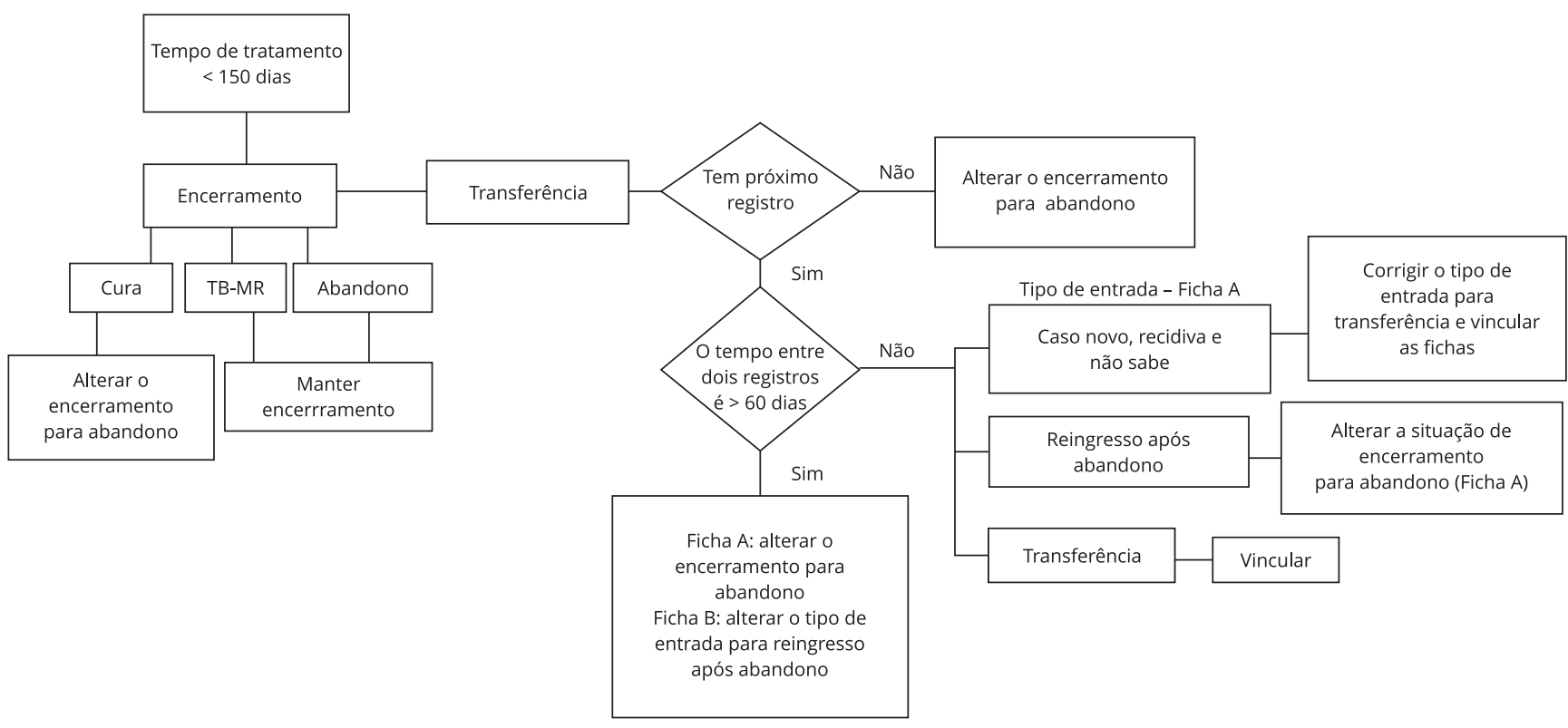

TB-MR: tuberculose multidrogarresistente.

realizar exames bacteriológicos; entre 150 e 270 dias, considerando que a presença de alguns agravos associados ocasionam o prolongamento do tratamento e, também, por ser considerado o período máximo para o encerramento oportuno no Sinan 1,14; e acima de 270 dias, fora do período esperado.

Para calcular o período entre notificações consecutivas do mesmo paciente, foram utilizadas as variáveis "data de encerramento" da ficha anterior e "data de notificação" da notificação subsequente. Para os pacientes com mais de uma notificação e com algum registro sem encerramento, considerou-se o tipo de entrada do registro subsequente, com o objetivo de qualificar aquela informação (Figura 4).

Quando o intervalo entre dois registros do mesmo paciente era maior do que 60 dias, considerouse abandono de tratamento. Ponderou-se um tempo maior do que 30 dias, de acordo com a definição de abandono 1, com o objetivo de não penalizar pacientes que estavam em tratamento autoadministrado. Quanto ao tempo de tratamento, foi calculado a partir da data de encerramento e da data do início do tratamento.

Registros que tinham sido encerrados por “cura”, mas que apresentavam uma nova notificação em um período menor do que 60 dias não foram corrigidos, pois concluiu-se que uma análise caso a caso seria mais apropriada. Esses registros foram classificados como "analisar posteriormente" (Figuras $1,2$ e 3$)$.

Considerou-se "óbito por TB" no Sinan aqueles que no SIM tinham menção na causa básica dos códigos A15 ao A19, da Classificação Internacional de Doenças - 10a revisão (CID-10). Quando não havia menção da TB na causa básica, considerou-se "óbito por outras causas”. O mesmo procedimento foi adotado quando encontrávamos no SIM um óbito de paciente com coinfecção TB-HIV, conforme orientação do Manual de Recomendações 1. Pacientes não encontrados no SIM, por meio do linkage, mas que estavam encerrados por óbito no Sinan-TB, não tiveram o encerramento alterado no Sinan-TB (Figura 5). 
Figura 2

Diagrama com os critérios para qualificação da base de dados do Sistema de Informação de Agravos de Notificação - Tuberculose (Sinan-TB) em pacientes com $\geq 150$ a $<270$ dias de tratamento.

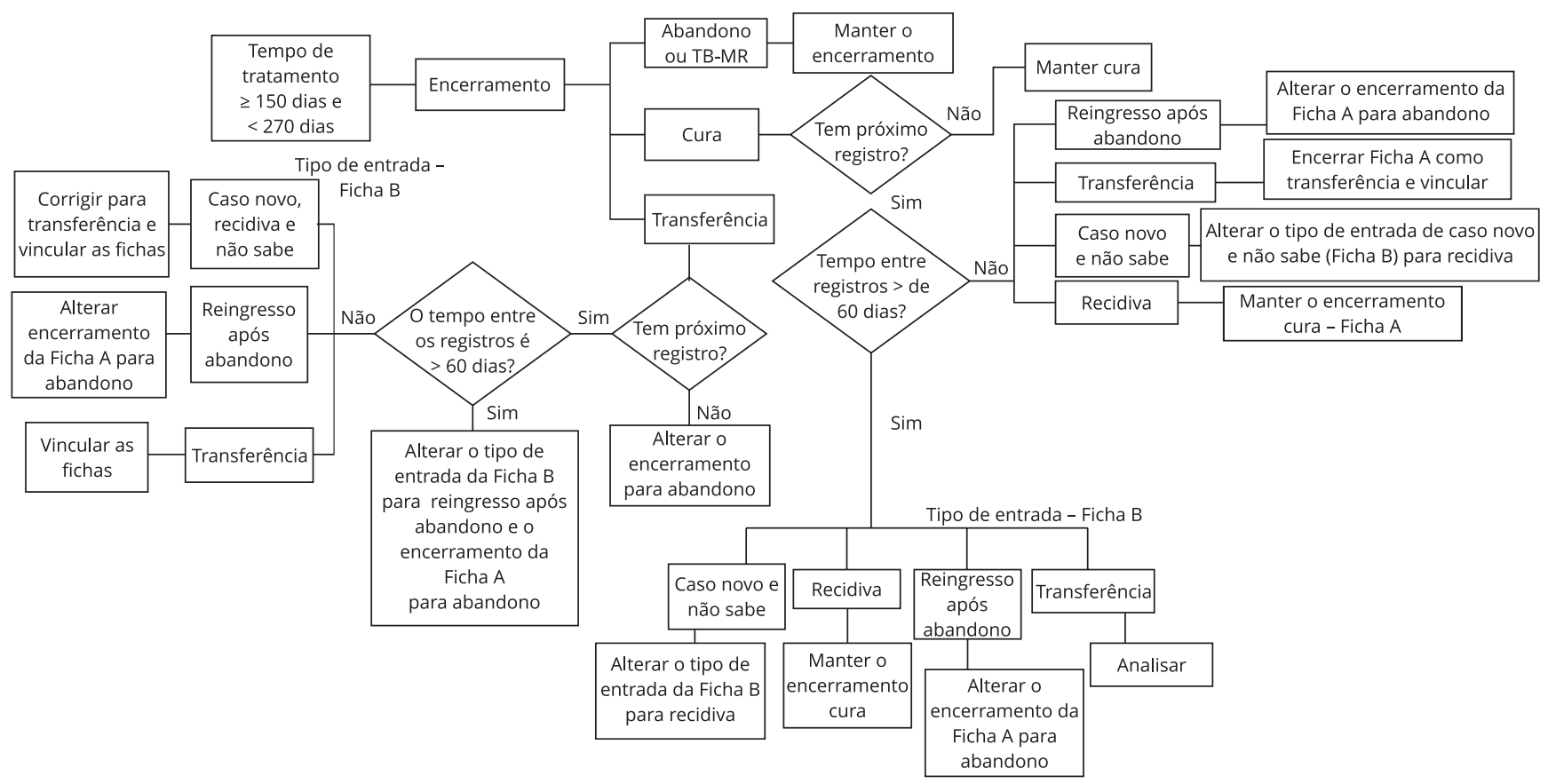

TB-MR: tuberculose multidrogarresistente.

Nas situações em que o encerramento era por "cura", "transferência” ou "ignorado/em branco", e o óbito tivesse ocorrido dentro do período de tratamento terapêutico básico (180 dias), a "situação de encerramento" era corrigida para óbito, segundo os critérios descritos anteriormente. Para análise de sensibilidade e definição do período para mudança dessa variável, utilizou-se também o período 180 a 270 e de 270 dias ou mais para a comparação (Figura 5).

Quando a variável "situação de encerramento" era alterada para óbito, a "data de encerramento" do último registro no Sinan-TB passava a ser a do óbito registrada no SIM. Esse critério também foi aplicado aos encerramentos por óbito em que havia divergência entre as datas do encerramento e do óbito.

Para os registros com "data de notificação" anterior a 2006, ponderou-se um período de cinco anos entre dois registros para alterar o "tipo de entrada”. Isso em decorrência da definição antiga 15, na qual o paciente era considerado como caso novo quando o intervalo entre os tratamentos fosse superior a cinco anos.

Os registros com apenas uma entrada no Sinan-TB, diagnosticados até 2012 e sem encerramento, foram encerrados por "abandono" (Figura 4). A "situação de encerramento" não foi alterada em pacientes que apresentaram diagnóstico da TB no ano de 2013 e encerraram em 2014 por transferência por considerar a possibilidade de atraso na atualização da informação.

Os encerramentos por TB multidrogarresistente (TB-MR) não sofreram correção, pois o protocolo de notificação desses casos orienta que após o diagnóstico de TB-MR o paciente seja notificado em outro sistema de informação específico para tratamentos especiais, o qual não foi analisado neste estudo. 
Figura 3

Diagrama com os critérios para qualificação da base de dados do Sistema de Informação de Agravos de Notificação - Tuberculose (Sinan-TB) em pacientes com $\geq 270$ dias de tratamento.

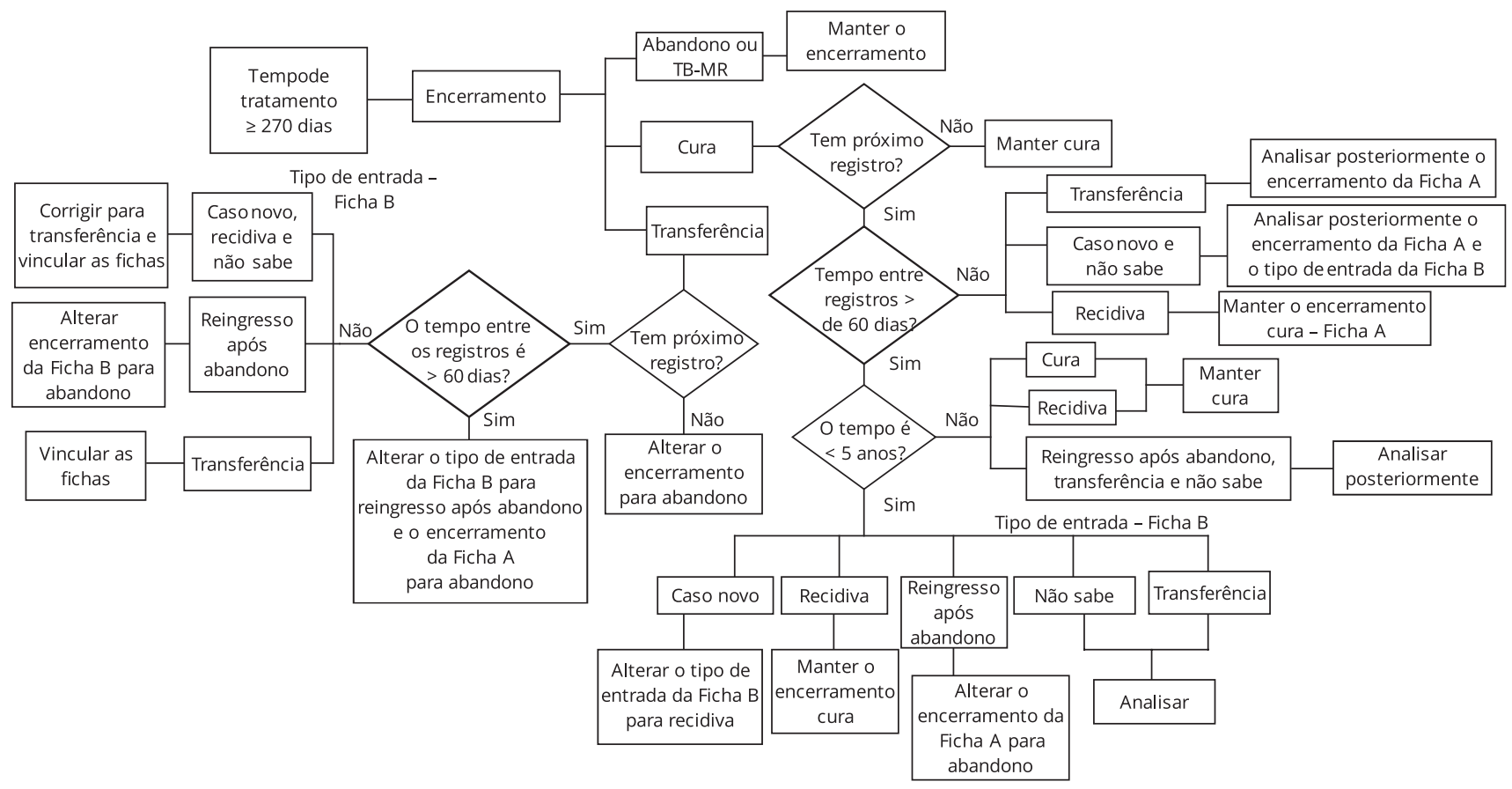

TB-MR: tuberculose multidrogarresistente.

Valendo-se da base única da aids, foi possível qualificar a variável "agravo associado aids" e "teste para HIV" no Sinan-TB. Todo paciente com "agravo associado aids" assinalado como "sim" tem, automaticamente, a variável "teste para HIV" assinalada como "positivo" 14. Considerou-se coinfecção quando o diagnóstico do HIV/aids ocorreu antes ou durante o tratamento terapêutico básico para a TB (Figura 6).

A correção da variável "agravo associado diabetes" considerou os óbitos ocorridos em até 270 dias após o diagnóstico da TB e que apresentaram, dentre as causas de morte, menção aos códigos da CID-10 referentes ao diabetes (E10.0 ao E14.9) (Figura 7).

\section{Análise dos dados}

Foi realizada análise descritiva das variáveis corrigidas, considerando-se os perfis anterior e posterior à qualificação da base de dados do Sinan-TB. A análise foi realizada considerando-se três opções de períodos de tratamento: menos de 150 dias, entre 150 e 270 dias e 270 e mais.

$\mathrm{Na}$ construção dos critérios para a qualificação das variáveis citadas, foram usados os seguintes materiais técnicos do Ministério da Saúde: Manual de Recomendações para o Controle da Tuberculose no Brasil 1 e Manual de Normas e Rotinas do Sinan 14.

Utilizou-se a scripting Structured Query Language (SQL) 16, por meio do software PostgreSQL versão 9.6.3 (https://www.postgresql.org/) 17, para a implantação dos critérios de qualificação. A análise descritiva foi realizada com o auxílio do pacote estatístico R versão 3.3.1 (http://www.r-project. org). O linkage probabilístico foi realizado com o auxílio do software OpenRecLink (http://reclink. sourceforge.net/) 18 . 


\section{Figura 4}

Diagrama com os critérios para qualificação da base de dados do Sistema de Informação de Agravos de Notificação Tuberculose (Sinan-TB) para registros sem encerramento.

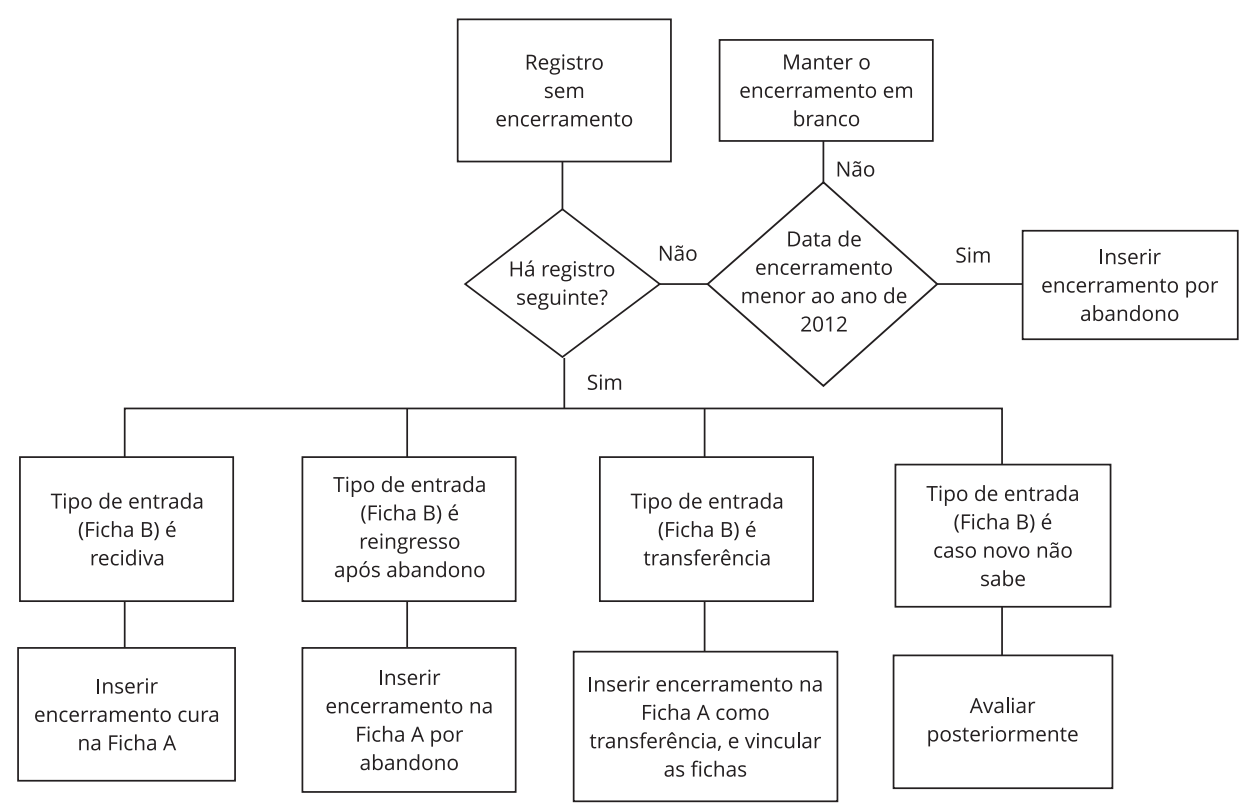

\section{Figura 5}

Diagrama com os critérios para qualificação da base de dados do Sistema de Informação de Agravos de Notificação Tuberculose (Sinan-TB) quanto à informação do óbito.

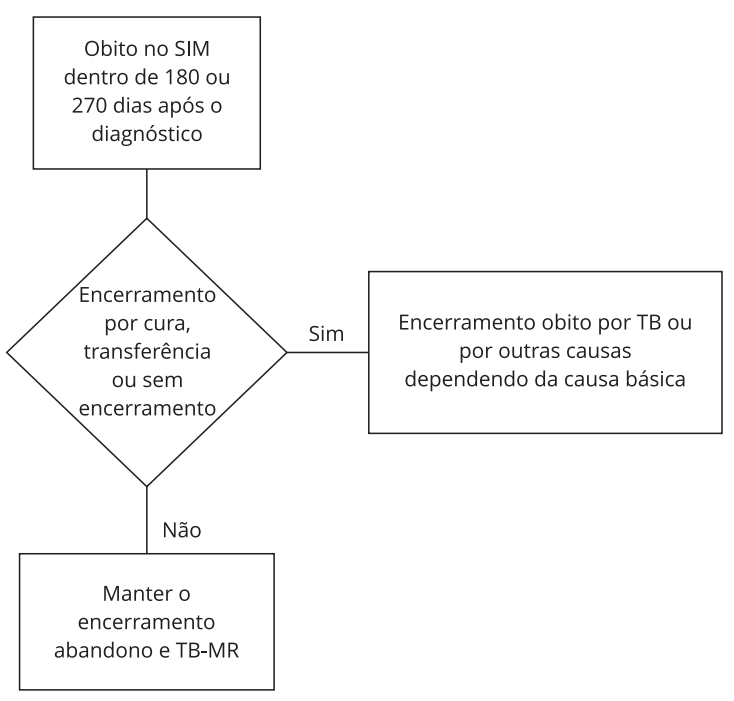

SIM: Sistema de Informações sobre Mortalidade; TB-MR: tuberculose multidrogarresistente. 


\section{Figura 6}

Diagrama com os critérios para qualificação da base de dados do Sistema de Informação de Agravos de Notificação Tuberculose (Sinan-TB) quanto à informação para HIV/aids.

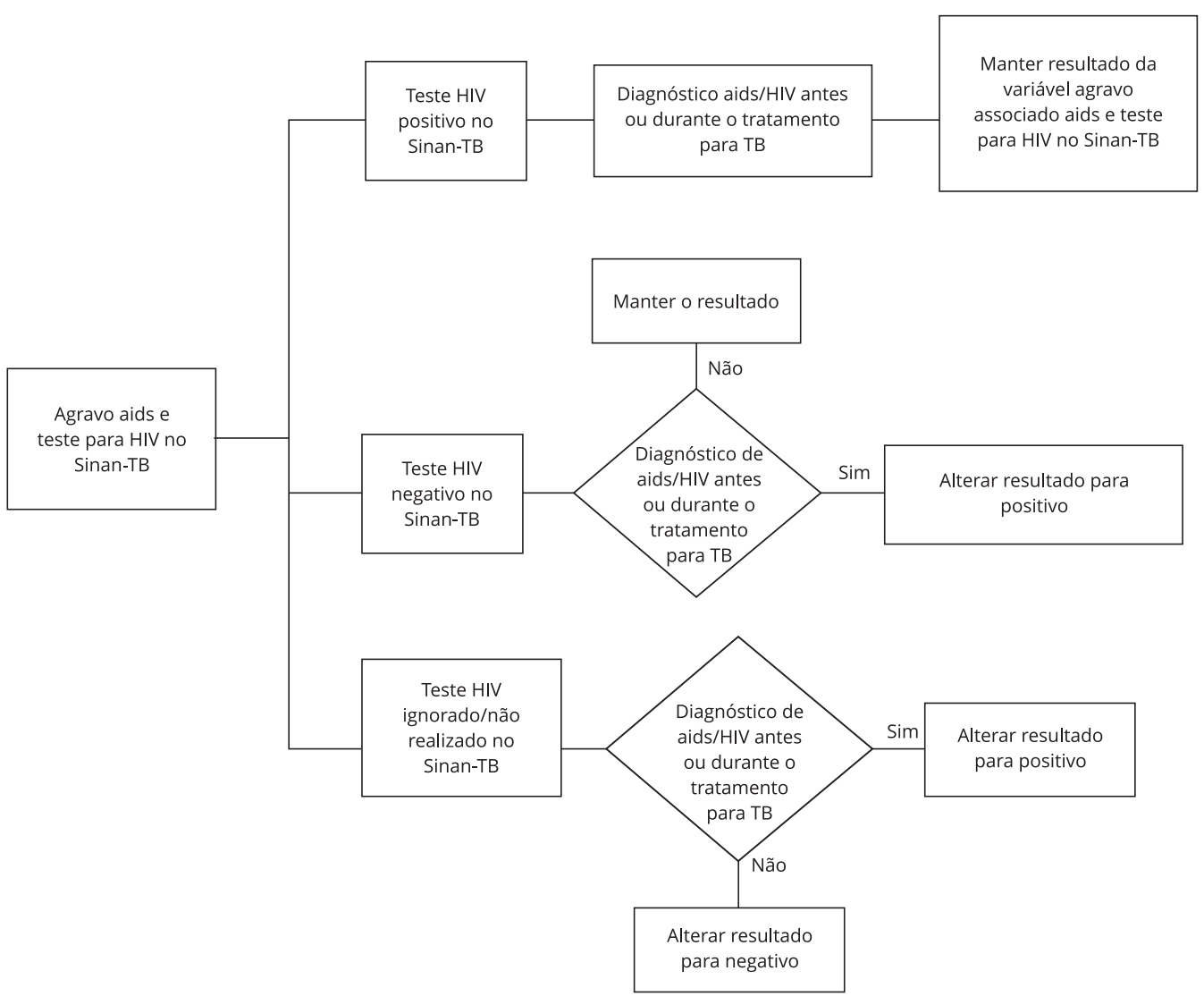

O trabalho foi aprovado pelo Comitê de Ética em Pesquisa do Instituto de Estudos em Saúde Coletiva, Universidade Federal do Rio de Janeiro (IESC/UFRJ), processo no CAAE 07537412.2.0000.5286, parecer no 896.897 e processo no CAAE 06760412.7.0000.5286, parecer no 141.013.

\section{Resultados}

Foram analisados 37.531 registros de pacientes notificados no Sinan-TB no período de 2009 a 2013, no Município do Rio de Janeiro.

Após aplicação dos critérios para qualificação das variáveis, a maior mudança ocorreu com relação à situação de encerramento "abandono" ( $\mathrm{n}=5.611 / 15 \%$ vs. 12.079/32,2\%), principalmente em função do acréscimo de "transferências" $(\mathrm{n}=3.139)$, dos encerramentos em branco $(\mathrm{n}=1.994)$ e da "cura" $(\mathrm{n}=1.331)$ (Tabelas 1 e 2). A diminuição observada no encerramento "cura" ocorreu principalmente por conta dos pacientes que encerraram o tratamento em menos de 150 dias (Tabela 1).

Houve redução expressiva dos registros sem encerramento, de 9,2\% para 3,5\%, e no encerramento por "transferência" a redução foi ainda mais expressiva, de 9,1\% para 0,7\% (Tabela 1).

A categoria "óbito por outras causas" apresentou um aumento significativo, e a categoria "óbito por TB", uma importante redução. Esse aumento da categoria "óbito por outras causas" se deve ao 
Diagrama com os critérios para qualificação da base de dados do Sistema de Informação de Agravos de Notificação Tuberculose (Sinan-TB) quanto à informação sobre diabetes.

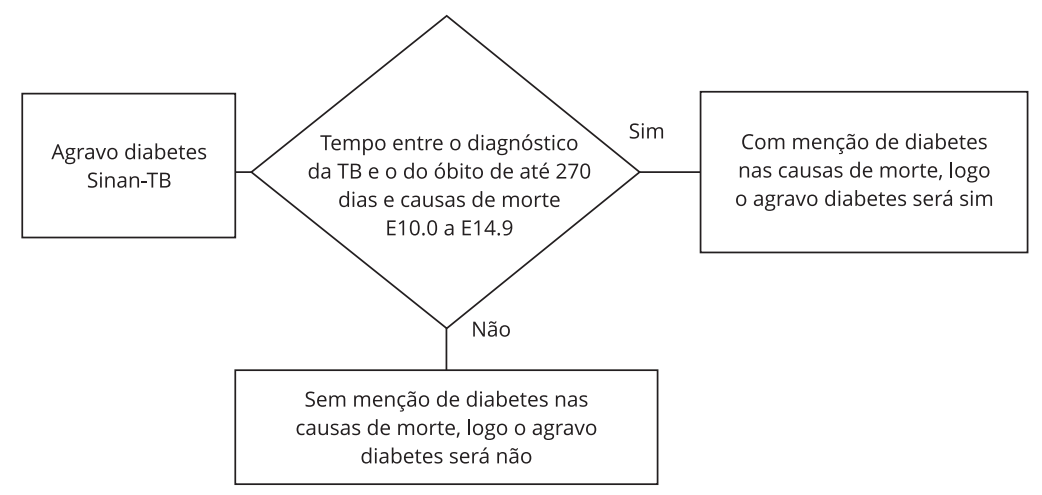

acréscimo de 677 registros da categoria "óbito por TB", de 72 sem encerramento, de 66 registros encerrados por "cura” e quatro por "transferência" (Tabela 2).

A análise de sensibilidade utilizando o intervalo de 180 dias e de 270 dias entre o início do tratamento e o óbito revelou um acréscimo de sete óbitos por TB (1.257 vs. 1.264) e de 56 óbitos por outras causas (1.711 vs. 1.767), quando utilizado o tempo de 270 dias (dado não mostrado).

A variável "tipo de entrada" sofreu discreta mudança, com leve diminuição no número de casos novos e aumento na categoria "reingresso após abandono", que recebeu 31 registros das categorias "recidiva” e "transferência” (Tabela 1).

Ao compararmos os resultados usando diferentes tempos de tratamento, percebe-se que no período menor de 150 dias todos os encerramentos por "cura" ( $n=928,9,7 \%)$ foram alterados para "abandono". No geral, os encerramentos por "óbito por TB" diminuíram e os encerramentos na categoria "óbito por outras causas" aumentaram; houve uma diminuição dos encerramentos por "transferência" e, consequentemente, aumento do encerramento por "abandono" em todos os tempos analisados (Tabela 1).

A qualificação da variável "agravo associado aids" valendo-se da base única da aids acarretou um aumento de $8,7 \%$ na categoria "sim" (11,1\% vs. 12,1\%) e uma diminuição na categoria "ignorado" de 2,2\% (37,6\% vs. 36,8\%). Quanto à categoria "não", praticamente não houve mudança. O campo "teste para HIV” aumentou 5,3\% (12,2\% vs. 12,8\%) (Tabela 3).

Quanto ao "agravo associado diabetes", houve um acréscimo de 54 registros na categoria "sim”, o que representou um aumento de 2,4\% (6,1\% vs. 6,3\%) (Tabela 3).

\section{Discussão}

A proposta de qualificação da base do Sinan-TB visou a minimizar inconsistências nos registros quanto aos campos "tipo de entrada", "situação de encerramento", "teste para HIV", "agravo associado aids" e "agravo associado diabetes", considerando a informação existente no próprio Sinan-TB, no SIM e na base única da aids. Esse procedimento é importante por oferecer aos programas de controle da TB dados mais fidedignos que subsidiarão a tomada de decisão.

Com a qualificação, foi evidenciado um percentual de abandono de tratamento muito acima do divulgado pelos dados oficiais, revelando uma situação ainda mais grave que pode ocasionar a permanência da transmissibilidade da doença ${ }^{19}$, possível aumento dos casos de resistência às drogas 20,21 e 
Tabela 1

Distribuição do "tipo de entrada" e da "situação de encerramento" antes e após a implementação dos critérios de qualificação para tempo de tratamento < 150 dias, $\geq 150$ a 270 dias e $\geq 270$ dias. Rio de Janeiro, Brasil, 2009 a 2013.

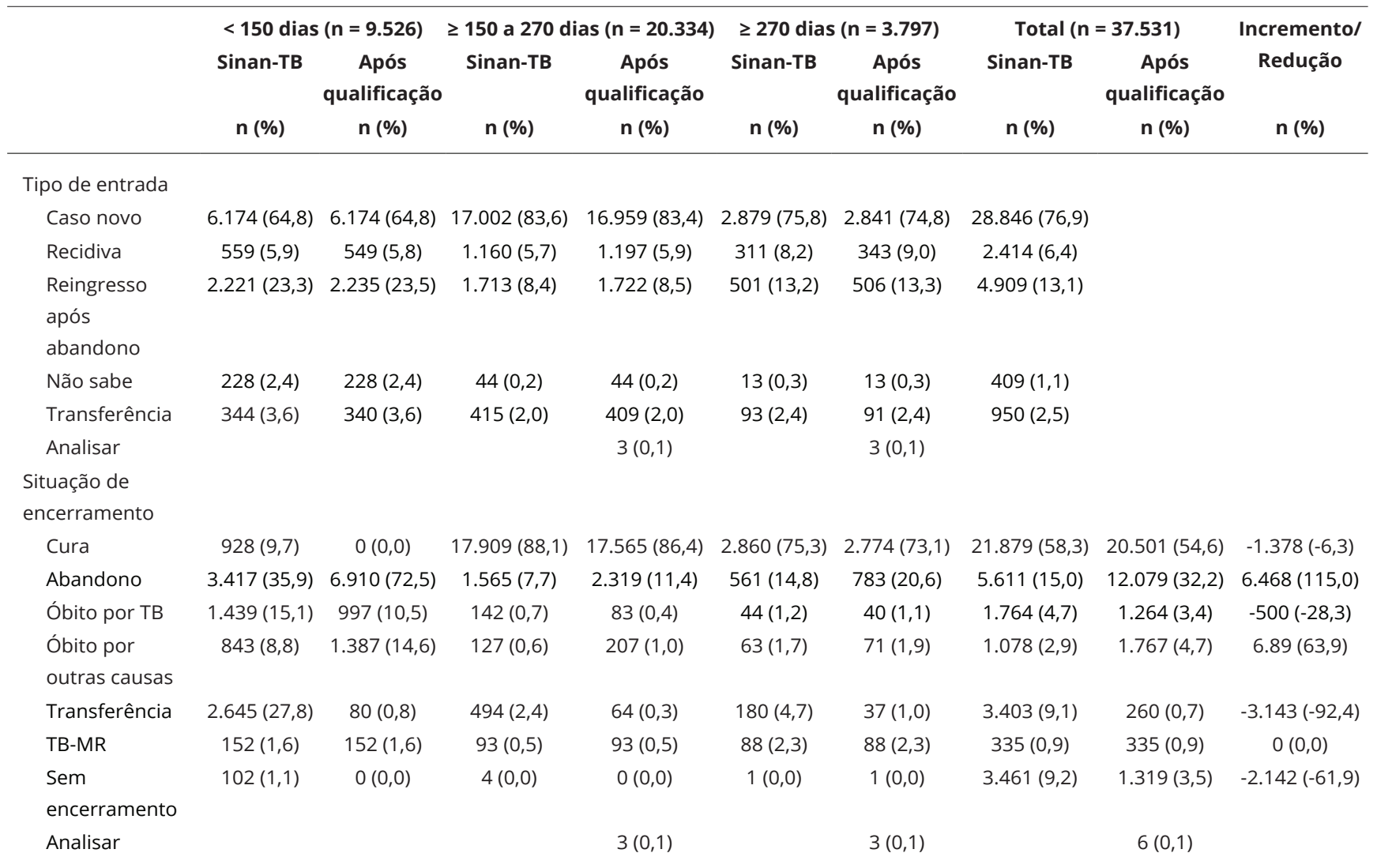

Sinan-TB: Sistema de Informação de Agravos de Notificação - Tuberculose; TB-MR: tuberculose multidrogarresistente.

Nota: Registros sem encerramento não foram incluídos por não constar a data de encerramento $(n=3.874)$. Os períodos $<150$ dias, $\geq 150$ a 270 dias e $\geq$ 270 dias referem-se ao tempo de tratamento.

\section{Tabela 2}

Distribuição da situação de encerramento no Sistema de Informação de Agravos de Notificação - Tuberculose (Sinan-TB) antes e após a qualificação. Rio de Janeiro, Brasil, 2009 a 2013.

\begin{tabular}{|c|c|c|c|c|c|c|c|c|}
\hline \multirow[t]{2}{*}{ Original } & \multicolumn{8}{|c|}{ Após qualificação } \\
\hline & Cura & Abandono & Óbito TB & $\begin{array}{l}\text { Óbito outras } \\
\text { causas }\end{array}$ & Transferência & TB-MR & $\begin{array}{c}\text { Sem } \\
\text { encerramento }\end{array}$ & Analisar \\
\hline Cura & 20.463 & 1.331 & 12 & 66 & 1 & 0 & 0 & 6 \\
\hline Abandono & 0 & 5.611 & 0 & 0 & 0 & 0 & 0 & 0 \\
\hline Óbito TB & 0 & 4 & 1.083 & 677 & 0 & 0 & 0 & 0 \\
\hline $\begin{array}{l}\text { Óbito outras } \\
\text { causas }\end{array}$ & 0 & 0 & 130 & 948 & 0 & 0 & 0 & 0 \\
\hline Transferência & 0 & 3.139 & 3 & 4 & 257 & 0 & 0 & 0 \\
\hline TB-MR & 0 & 0 & 0 & 0 & 0 & 335 & 0 & 0 \\
\hline Sem encerramento & 38 & 1.994 & 36 & 72 & 2 & 0 & 1.319 & 0 \\
\hline
\end{tabular}

TB-MR: tuberculose multidrogarresistente. 


\section{Tabela 3}

Distribuição das variáveis "agravo associado aids", "teste para HIV" e "agravo associado diabetes" antes e após a qualificação da base. Rio de Janeiro, Brasil, 2009 a 2013.

\begin{tabular}{|c|c|c|c|c|c|c|}
\hline & \multicolumn{2}{|c|}{ Sinan-TB } & \multicolumn{2}{|c|}{ Após qualificação } & \multicolumn{2}{|c|}{ Incremento/Redução } \\
\hline & $\mathbf{n}$ & $\%$ & n & $\%$ & $\mathbf{n}$ & $\%$ \\
\hline \multicolumn{7}{|c|}{ Agravo associado aids } \\
\hline Sim & 4.185 & 11,2 & 4.550 & 12,1 & 365 & 8,7 \\
\hline Não & 19.236 & 51,3 & 19.176 & 51,1 & -60 & $-0,3$ \\
\hline Ignorado & 14.110 & 37,6 & 13.805 & 36,8 & -305 & $-2,2$ \\
\hline \multicolumn{7}{|l|}{ Teste para HIV } \\
\hline Positivo & 4.563 & 12,2 & 4.803 & 12,8 & 240 & 5,3 \\
\hline Negativo & 18.103 & 48,2 & 18.133 & 48,3 & 30 & 0,2 \\
\hline Não realizado & 14.865 & 39,6 & 14.595 & 38,9 & -270 & $-1,8$ \\
\hline \multicolumn{7}{|c|}{ Agravo associado diabetes } \\
\hline Sim & 2.297 & 6,1 & 2.351 & 6,3 & 54 & 2,4 \\
\hline Não & 26.493 & 70,6 & 26.461 & 70,5 & -32 & $-0,1$ \\
\hline Ignorado & 8.741 & 23,3 & 8.719 & 23,2 & -22 & $-0,3$ \\
\hline
\end{tabular}

Sinan-TB: Sistema de Informação de Agravos de Notificação - Tuberculose.

recidiva da doença, em função da entrega dos medicamentos para 30 dias a pacientes com cinco meses de tratamento, porém, sem a supervisão de um profissional de saúde e da realização de exames que confirmem a cura 22 .

Os critérios elaborados privilegiaram a manutenção do abandono em vez de alterar o encerramento para óbito, quando este evento houvesse ocorrido. O objetivo foi preservar a informação do abandono por se tratar de uma informação importante, principalmente por ser uma circunstância intermediária. Ao alterar essa informação para óbito, mascararíamos uma informação, que pode ser modificada por ações que favoreçam a adesão ao tratamento. Assim sendo, recomendamos que o Sinan-TB incorpore um campo que considere essa condição intermediária com o objetivo de instrumentalizar a vigilância, mensurar o verdadeiro percentual de abandono de tratamento e avaliar o percentual de óbitos associados ao abandono prévio.

Decidiu-se por alterar o encerramento "cura", "transferência” ou em branco para óbito, quando este evento ocorresse em até 270 dias em vez de 180 dias após o início do tratamento, em concordância com outros estudos 2,5 e, também, por ser o período recomendado para o encerramento oportuno no Sinan 14. Dessa forma, foi incluído um número maior de pessoas que morreram por TB e que, por algum motivo, não tiveram o encerramento em até seis meses após o início do tratamento.

A principal variação no encerramento óbito ocorreu na categoria "óbito por outras causas", a qual recebeu registros, principalmente da categoria “óbito por TB”. Rocha et al. 2, em um estudo sobre a confiabilidade do encerramento no Sinan-TB, demonstraram que 61,5\% dos registros encerrados por “óbito por TB" não apresentavam menção da TB nas causas de morte ou a TB era a causa associada, ou seja, deveriam ser classificados como "óbito por outras causas" 1. Provavelmente, o erro de preenchimento ocorre por conta do desconhecimento dos profissionais de saúde sobre a causa básica de morte, os quais pressupõem que a TB seja a causa básica por ter a morte ocorrido durante o tratamento.

De forma a contribuir com esses resultados e reforçar a importância do relacionamento entre as bases de dados, Oliveira et al. 3 ao compararem os registros encerrados por óbito por/com TB e o SIM, concluíram que 43,7\% estavam encerrados corretamente como óbito, e 19,7\% e 20,1\% tinham como encerramento o abandono do tratamento e a cura, respectivamente. Após o linkage, para 2005, o percentual de encerramento por óbito aumentou de 6,5 para 6,9\%. Em 2006, esse valor aumentou de 5,6 para $6,4 \%$ e o percentual de registros sem desfecho de tratamento reduziu de $14 \%$ para $13,7 \%$. 
A qualificação da base de dados possibilitou a redução significativa dos encerramentos por "transferência”. Entretanto, um fator que pode ter contribuído para o percentual de transferência não ter diminuído ainda mais foi a não vinculação dos registros após a execução dos critérios. Esse procedimento visa a unir dois ou mais registros de um paciente durante o mesmo tratamento, nas situações em que a pessoa é transferida para dar continuidade ao tratamento em outra unidade de saúde 14.

O relacionamento entre as bases de dados do Sinan-TB e a base única da aids aumentou a informação no Sinan-TB sobre a prevalência da coinfecção TB-HIV. Esse resultado foi similar ao encontrado em estudos realizados por Magno et al. 23 e por Carvalho et al. 24 , nos quais a prevalência da coinfecção aumentou de $6,7 \%$ para $7,7 \%$ e de $6 \%$ para $8,4 \%$, respectivamente, após o relacionamento entre as bases de dados. Ressalta-se, mais uma vez, a relevância da integração entre as bases de dados e também entre as coordenações das respectivas doenças com o objetivo de qualificar essa informação para a vigilância de ambos os agravos.

O adequado preenchimento das variáveis "agravo associado aids" e "teste para HIV" é importante, pois com base nelas calcula-se a prevalência de coinfecção no país, o que auxiliará o planejamento e o monitoramento das ações de prevenção e controle. Lírio et al. 6 demonstraram que, apesar da completude do campo "HIV" do Sinan-TB ser maior do que 50\%, mais da metade dos registros estavam preenchidos como "em andamento" ou "não realizado". O campo "HIV" preenchido como "em andamento" não apresenta nenhuma informação consistente para a vigilância da TB. É necessário que essa informação seja atualizada oportunamente no Sinan-TB e, se positivo, o paciente também deve ser notificado, no Sinan, para HIV/aids.

A utilização da base de dados do SIM permitiu uma discreta qualificação da variável "agravo associado diabetes”. Cascão et al. 25 apontam a subnotificação do diabetes nas declarações de óbito, o que pode estar ligado à pequena alteração observada.

Uma limitação do estudo é o preenchimento da data de encerramento devido ao seu caráter estritamente administrativo, o que pode ter influenciado, principalmente na qualificação da variável "situação de encerramento" e na presença de tempos de tratamentos negativos, que podem ser reflexo de erro no preenchimento.

Podemos citar também outras limitações relacionadas ao uso de dados secundários, como a falha no processo de linkage, erros de digitação e de classificação dos registros utilizados. Consideramos, ainda, a possibilidade de o encerramento estar em branco devido ao paciente ter sido transferido espontaneamente para dar continuidade ao tratamento em outro município, e por isto ter sido reclassificado erroneamente como encerramento por "abandono".

Destaca-se o uso do relacionamento probabilístico e da SQL na qualificação da informação proveniente do Sinan para a vigilância da TB, e a potencial replicabilidade do método proposto, por estar baseado nas recomendações do Ministério da Saúde para o preenchimento do Sinan-TB e classificação dos casos. O próximo desafio será a atualização dos critérios para a inclusão das variáveis novas, como uso de terapia antirretroviral e tipo de entrada pós-óbito que foram acrescentadas na versão 5.0 do Sinan-Net, que ocorreu em 2014. 


\section{Colaboradores}

M. S. Rocha e R. S. Pinheiro contribuíram com a concepção e projeto, análise e interpretação dos dados, redação e revisão crítica relevante do conteúdo intelectual e aprovação final da versão a ser publicada; são responsáveis por todos os aspectos do trabalho na garantia da exatidão e integridade de qualquer parte da obra. G. P. Oliveira, C. M. Coeli e V. Saraceni contribuíram com a concepção e projeto, análise e interpretação dos dados, redação e revisão crítica relevante do conteúdo intelectual e aprovação final da versão a ser publicada. L. C. T. Guillen contribuiu com a concepção e projeto, análise e interpretação dos dados e aprovação final da versão a ser publicada.

\section{Informações adicionais}

ORCID: Marli Souza Rocha (0000-0003-23580848); Gisele Pinto de Oliveira (0000-0003-06895055); Luis Carlos Torres Guillen (0000-00015246-733X); Claudia Medina Coeli (0000-00031757-3940); Valéria Saraceni (0000-0001-73606490); Rejane Sobrino Pinheiro (0000-0002-33613626).

\section{Agradecimentos}

Ao Conselho Nacional de Desenvolvimento Científico e Tecnológico (CNPq; processos no 310173/2015-9 e no 305545/2015-9), Coordenação de Aperfeiçoamento de Pessoal de Nível Superior (Capes), Fundação de Amparo à Pesquisa do Estado do Rio de Janeiro (FAPERJ; processo no E/26-203.195/2015) e à Secretaria de Vigilância em Saúde, Ministério da Saúde/Fundação Nacional de Saúde (TC 234/2012) pelo apoio financeiro.

\section{Referências}

1. Departamento de Vigilância Epidemiológica, Secretaria de Vigilância em Saúde, Ministério da Saúde. Manual de recomendações para o controle da tuberculose no Brasil. Brasília: Ministério da Saúde; 2011.

2. Rocha MS, Aguiar FP, Oliveira GP, Saraceni V, Coeli CM, Pinheiro RS. Confiabilidade do desfecho do tratamento usando linkage de bases de dados para a tuberculose. Cad Saúde Colet (Rio J.) 2015; 23:150-6.

3. Oliveira GP, Pinheiro RS, Coeli CM, Codenotti SB, Barreira D. Linkage entre SIM e SINAN para a melhoria da qualidade dos dados do sistema de informação da tuberculose: a experiência nacional. Cad Saúde Colet (Rio J.) 2010; 18:107-11.

4. Medeiros D, Sucupira ED, Guedes RM, Costa AJL. Análise da qualidade das informações sobre tuberculose no Município de Belford Roxo, Rio de Janeiro, 2006 a 2008. Cad Saúde Colet (Rio J.) 2012; 20:146-52.

5. Bartholomay P, Oliveira GP, Pinheiro RS, Vasconcelos AMN. Melhoria da qualidade das informações sobre tuberculose a partir do relacionamento entre bases de dados. Cad Saúde Pública 2014; 30:2459-70.

6. Lírio M, Santos NP, Passos LAR, Kritski AL, Galvão-Castro B, Grassi MFR. Completude das fichas de notificação de tuberculose nos municípios prioritários da Bahia para controle da doença em indivíduos com HIV/AIDS. Ciênc Saúde Colet 2015; 20:1143-8.

7. Santos NP, Lírio M, Passos LAR, Dias JP, Kritski AL, Galvão-Castro B, et al. Completude das fichas de notificações de tuberculose em cinco capitais do Brasil com elevada incidência da doença. J Bras Pneumol 2013; 39:221-5.

8. Malhão TA, Oliveira GP, Codennoti S, Moherdaui F. Avaliação da completitude do Sistema de Informação de Agravos de Notificação da Tuberculose, Brasil, 2001-2006. Epidemiol Serv Saúde 2010; 19:245-56.

9. GBD 2016 Causes of Death Collaborators. Global, regional, and national age-sex specific mortality for 264 causes of death, 1980-2016: a systematic analysis for the Global Burden of Disease Study 2016. Lancet 2017; 390:1151210.

10. Oliveira GP, Bierrenbach ALS, Camargo Jr. KR, Coeli CM, Pinheiro RS. Acurácia das técnicas de relacionamento probabilístico e determinístico: o caso da tuberculose. Rev Saúde Pública 2016; 50:49.

11. Bierrenbach AL, de Oliveira GP, Codenotti S, Gomes AB, Stevens AP. Duplicates and misclassification of tuberculosis notification records in Brazil, 2001-2007. Int J Tuberc Lung Dis 2010; 14:593-7.

12. Ministério da Saúde. Boletim Epidemiológico HIV/AIDS 2016; Ano V, n. 1. http:// www.aids.gov.br/sites/default/files/anexos/ publicacao/2016/59291/boletim_2016_1_ pdf_16375.pdf. 
13. Santos ML. Fatores associados à subnotificação de TB e AIDS, durante os anos de 2001 a 2010, a partir do Sinan [Dissertação de Mestrado]. Recife: Centro de Pesquisas Aggeu Magalhães, Fundção Oswaldo Cruz; 2014.

14. Ministério da Saúde. Sistema de Informação Agravos de Notificação - SinanNET: manual do sistema. Brasília: Ministério da Saúde; 2007.

15. Comitê Técnico-científico de Assessoramento à Tuberculose; Comitê Assessor para Co-infeccção HIV-Tuberculose. Guia de vigilância epidemiológica. Brasília: Ministério da Saúde; 2002.

16. Date CJ. Introdução a sistemas de banco de dados. v. 1. Rio de Janeiro: Editora Campus; 2004.

17. Simkovics S. Enhancement of the ANSI SQL Implementation of PostgreSQL. Viena: Department of Information Systems, Vienna University of Technology; 1998.

18. Camargo Jr. KR, Coeli CM. Going open source: some lessons learned from the development of OpenRecLink. Cad Saúde Pública 2015; 31:257-63.

19. Chirinos NEC, Meirelles BHC. Fatores associados ao abandono do tratamento da tuberculose: uma revisão integrativa. Texto \& Contexto Enferm 2011; 20:599-606.
20. Faustini A, Hall AJ, Perucci CA. Risk factors for multidrug resistant tuberculosis in Europe: a systematic review. Thorax 2006; 61:158-63.

21. Garrido MS, Buher-Sékula S, Souza AB, Oliveira GP, Antunes IA, Mendes JM, et al. Multidrug-resistant tuberculosis in the Amazonas State, Brazil, 2000-2011. Int J Tuberc Lung Dis 2015; 19:531-6.

22. Oliveira HB, Moreira Filho DC. Recidivas em tuberculose e seus fatores de risco. Rev Panam Salud Pública 2000; 7:232-41.

23. Magno ES, Saraceni V, Souza AB, Magno RS, Saraiva MGG, Bührer-Sékula S. Fatores associados à coinfecção tuberculose e HIV: o que apontam os dados de notificação do Estado do Amazonas, Brasil, 2001-2012. Cad Saúde Pública 2017; 33:e0019315.

24. Carvalho CN, Dourado MIC, Bierrenbach ALS. Subnotificação da comorbidade tuberculose e AIDS: uma aplicação do método de linkage. Rev Saúde Pública 2011; 45:548-55.

25. Cascão AM, Costa AJL, Kale PL. Qualidade da informação sobre mortalidade numa coorte de diabéticos - Estado do Rio de Janeiro, 2000 a 2003. Rev Bras Epidemiol 2012; 15:134-42. 


\section{Abstract}

Brazil's Information System on Diseases of Notification (Sinan) is the main tool used by tuberculosis (TB) control programs to assess control measures and TB incidence. This requires data from the system that are reliable and accurate, among other features. The study thus aimed to upgrade the entry variables, condition at closure, HIV testing, AIDS-related illness, and diabetes. Linkage was performed between the Sinan-TB database, the Mortality Information System (SIM), and the single AIDS database for the city of Rio de Janeiro, Brazil. Criteria for upgrading the variables were based on technical materials on $T B$ and the Sinan database and were implemented in a script in Structured Query Language (SQL). There was a $115 \%$ increase in treatment dropout due to the decrease in transfers, records without closure, and patients closed due to cure in less than 150 days. There was a $2.4 \%$ increase in records with diseases associated with diabetes in the affirmative category after using data from the SIM. For the variables HIV testing and AIDS-associated illness, the increases were $5.3 \%$ and $8.7 \%$, respectively, when the information in the AIDS database was considered. In conclusion, upgrading the Sinan-TB database through integration with other information systems improved the data's quality for decisionmaking on TB control.

Information Systems; Medical Record Linkage; Tuberculosis; Data Accuracy; Database

\section{Resumen}

El Sistema de Información de Enfermedades de Notificación obligatoria (Sinan) es la principal herramienta utilizada por los programas de control de la tuberculosis (TB), para evaluar las acciones de control y la tasa de incidencia de la enfermedad. Para tal fin, es necesario que los datos provenientes de este sistema sean, entre otros aspectos, fiables y precisos. De esta forma, el objetivo de este estudio fue cualificar las variables: tipo de entrada, situación de cierre, test para VIH, enfermedad asociada al SIDA y diabetes. Se realizó una vinculación entre la base de datos del Sinan-TB, del Sistema de Información sobre Mortalidad (SIM) y la base única del SIDA del municipio de Río de Janeiro, Brasil. Los criterios para la cualificación de las variables están fundamentados en materiales técnicos sobre la TB y sobre el Sinan, y fueron implementados en un script con Structured Query Language (SQL). Se observó un aumento de un $115 \%$ en el abandono del tratamiento, a consecuencia de la disminución de transferencias, de los registros sin cierre $y$ de pacientes internados por tratamiento menos de 150 dias. Hubo un incremento de un 2,4\% de los registros con enfermedad asociada a la diabetes en la categoría SIM, tras utilizar la información contenida en el SIM. Para las variables test del VIH y enfermedad asociada al SIDA el incremento fue de un 5,3\% y un 8,7\%, respectivamente, considerando la información contenida en la base de datos del SIDA. Se concluyó que la cualificación de la base de datos del Sinan-TB, valiéndose de la integración con otros sistemas de información, perfeccionó la calidad de la información en la toma de decisiones para el control de la TB.

Sistemas de Información; Registro Médico

Coordinado; Tuberculosis; Exactitud de

los Datos; Base de Datos
Recebido em 13/Abr/2018

Versão final reapresentada em 15/Mai/2019

Aprovado em 23/Set/2019 